\title{
UN GRAIN DE SABLE DANS L'ENGRENAGE DU SYSTÈME JURIDIQUE. LES JUSTICIABLES NON REPRÉSENTÉS: PROBLÈMES OU SYMPTÔMES?*
}

\author{
Emmanuelle Bernheim** \\ Richard-Alexandre Laniel $^{* * *}$
}

Les justiciables non représentés sont de plus en plus nombreux à tenter de faire valoir leurs droits, y compris devant le plus haut tribunal du pays, ce qui n'est pas sans conséquences, tant pour les acteurs du système juridique que pour les justiciables euxmêmes.

Dans le présent article, les auteurs s'intéressent au discours doctrinal sur le phénomène de la non-représentation. Ils constatent dans un premier temps que les justiciables non représentés sont généralement tenus seuls responsables de la situation problématique provoquée par leur présence. Ils démontrent dans un deuxième temps comment l'ensemble des solutions proposées vise d'abord le maintien des conditions "normales » de fonctionnement du processus judiciaire, sans proposer de réflexion holistique ou systématique sur la question. Ils explorent finalement l'idée selon laquelle les fondements paradigmatiques de la raison juridique seraient les principaux obstacles à l'absence de réflexion globale et à l'incapacité de mettre en place efficacement des changements majeurs.

The number of self-represented litigants [SRL] that are trying to defend by themselves their rights are increasing constantly, even in the highest tribunals. This situation is not without consequences for both the actors of the legal system and the SRL themselves. In the last years, many initiatives have been taken in order to contain the negative effects of the presence of SRL in the system.

In this article, the authors are analysing the discourse coming from the legal doctrine on the phenomenon of self-representation. First of all, they find that SRL are generally held as the only responsible for the annoying consequences provoked by their very presence. Secondly, they demonstrate that the majority of the solutions submitted in the literature are meant to maintain the normal functioning of the legal system, without submitting any holistic or systematic solutions. Finally, they consider the possibility that it would be the paradigmatic basis of the legal thinking that is the principal barrier for a global assessment of the problematic and for the implementation of efficient solutions.

Trop souvent, le plaideur qui se représente lui-même est considéré comme un problème pour les juges et pour le système judiciaire plutôt que comme une personne pour qui le

\footnotetext{
* Cette recherche a été rendue possible grâce au soutien financier du Fond québécois de recherche Société et culture (programme établissement de nouveaux professeur-chercheurs) et du Conseil de recherche en sciences humaines du Canada (bourse de maîtrise).

** Professeure, département des sciences juridiques, Université du Québec à Montréal.

*** B.A., LL.B., étudiant à la maîtrise.
} 
système de justice civile existe. Le véritable problème, cependant, c’est que le système judiciaire et ses procédures sont toujours aussi inaccessibles et incompréhensibles pour les gens ordinaires ${ }^{1}$.

En Amérique du Nord ${ }^{2}$, le nombre de justiciables non représentés ${ }^{3}[J N R]$ devant les tribunaux - civils, criminels et administratifs ainsi qu'à tous les niveaux d'instance - ne cesse d'augmenter ${ }^{4}$. Bien que le phénomène ne soit pas nouveau, il semble qu'il connaisse une certaine recrudescence depuis une vingtaine d'années ${ }^{5}$. Au Québec, en matière familiale, 42 pour cent des dossiers concernent au moins un JNR $^{6}$. Seraient en cause les coûts excessifs de la justice et le bas niveau des plafonds d'admissibilité aux

1 Lord Woolf, M.R., Access to Justice - Interim Report to the Lord Chancellor on the civil justice system in England and Wales, Londres, 1996, para 2 (traduit par Trevor C. W. Farrow, Diana Lowe, Badley Albrecht, Heather Manweiller et Martha Simmons, Répondre aux besoins des PNRA dans le système canadien de justice, Livre blanc préparé à l'intention de l'Association des administrateurs judiciaires du Canada, Toronto et Edmonton, 2012, p 34).

2 Bien que notre réflexion s'inscrive dans le contexte québécois, le peu de données disponibles ne nous permet pas d'y circonscrire notre analyse. Nous nous reporterons donc à la situation au Québec en fonction de l'information disponible.

3 Nous préférons parler de « justiciables non représentés » $[J N R]$ plutôt que de « justiciables se représentant eux-mêmes » pour refléter le fait qu'il s'agit rarement d'un choix (Nicholas Bala et Rachel Birnbaum, « Family litigants without lawyers - Study documents growing challenges for the justice system » (2011) The lawyers weekly 31 (12), pp 9-11; Farrow, Lowe, Albrecht, Manweiller et Simmons, supra note 1 à la p 4), malgré ce que donne à penser une certaine littérature (par ex. Maria De Michele, «Seul devant la Cour », dans Barreau du Québec, Congrès du Barreau : À la mesure du panorama, juin 2010, pp 3 et ss [Seul]). Voir le blogue de Julie Macfarlane, Three Hard Realities Shaping the Self-Rep Experience: and what the Courts say about this, <drjuliemacfarlane.wordpress.com/2013/10/13/three-hardrealities-shaping-the-self-rep-experience-and-what-the-courts-say-about-this/> (consulté le 21 octobre 2013):

I have no choice - I am unrepresented, not self-represented. It's not that I think I can do this better than a lawyer. I have no choice. I don't have \$350 an hour to pay a lawyer. [...]

Despite the overwhelming evidence that money is the primary factor in self representation, and the numerous testimonies of SRL's to this point, judges continue to tell them - sometimes kindly and solicitously, sometimes disdainfully and contemptuously, that they "should" retain counsel to represent them.

4 Julie Macfarlane parle d'une « hausse dramatique » («dramatic increases ») : The National Self-Represented Litigants Project : Identifying and Meeting the Needs of Self-Represented Litigants, Rapport de recherche présenté aux fondations du droit de l'Ontario, de l'Alberta et de la Colombie-Britannique, mai 2013, p 15 [The National]. Voir également : Michel Tétrault, «Éthique et déontologie en droit de la famille. Le litige familial, la déontologie et l’Éthique : mais qui est contre la vertu ? », dans Barreau du Québec, Congrès du Barreau : À la mesure du panorama, juin 2010, p 204; Rachel Birnbaum et Nicholas Bala, «Views of Ontario Lawyers on family litigants without representation » (2012) RD UN-B 63, pp 99-124; Deborah J. Chase, « Pro Se Justice and Unified Family Court » (2003-02) Fam LQ 37, pp 404-05.

5 Steven K. Berenson, « A Family Law Residency Program ?: A Modest Proposal in Response to the Burdens created by Self-Represented Litigants in Family Court » (2001-02) Rutgers LJ 33, pp 105, 107-12; Elisabeth Richardson, Tania Sourdin et Nerida Wallace, Self-Represented Litigants: Literature Review, Australian Center for Court and Justice System Innovation, Monash University, 2012, p 13; Chase, ibid à la p 404; Macfarlane, ibid à la p 15; Association du Barreau canadien, Atteindre l'égalité devant la justice : une invitation à l'imagination et à l'action - Justice pour tous, trouver l'équilibre, Ottawa 2013.

$6 \quad$ Pierre-Claude Lafond, L'accès à la justice civile au Québec. Portrait général, Cowansville, Yvon Blais, 2012, p 55. En 2010, 25 pour cent des demandes déposées à la Cour suprême du Canada émanaient de JNR (Cour suprême du Canada, Budget des dépenses 2010-2011 - Un rapport sur les plans et les priorités, Ottawa, 2010). Voir également : Anne-Marie Langan, « Threatening the Balance of the Scales of Justice: Unrepresented Litigants in the Family Courts of Ontario » (2005) Queen's LJ 30, para 7. 
services d'aide juridique ${ }^{7}$. Le fait de se représenter soi-même devant les tribunaux - bien qu'étant un droit prévu au Code de procédure civile $e^{8}$ - est donc le plus souvent contextuel et non le fruit d'une décision délibérée.

Une telle situation n'est pas sans conséquence sur le déroulement du processus judiciaire et sur l'issue des litiges. La littérature fait état notamment de coûts globaux plus élevés ${ }^{9}$, de l'allongement des procédures et du prolongement des audiences ${ }^{10}$ et du fait que la protection des droits n'est pas forcément assurée $^{11}$. Les JNR ne comprendraient pas ce qui se passe avant et pendant les audiences et seraient mal préparés. Démunis, émotifs et « dépassés par la nature technique et la précision du processus judiciaire " ${ }^{12}$, ils ne parviendraient pas à mettre en œuvre une stratégie avantageuse; les décisions judiciaires leur seraient généralement défavorables ${ }^{13}$.

Dans un système où les rôles de tous les participants sont prédéterminés et interdépendants, indispensables au fonctionnement et à l'efficacité de l'ensemble, où une procédure prévoit tant la forme des processus décisionnels que leur substance, tout changement, même mineur, en perturbe tant l'efficacité que l'intégrité et la légitimité. La structure normative interne au système - relative tant au langage et aux référents qu'aux modalités de participation et aux paramètres d'intégration de l’information externe - établit et garantit la prévisibilité des rapports et des décisions. Cette structure normative prévoit notamment la production de faits signifiants qui, par leur interaction, contribuent à la fois à la reproduction et à l'atteinte des objectifs poursuivis par le système.

Autopoietic systems are systems that are defined as unities as networks of productions of components that recursively, through their interactions, generate and realize the network that produce them and constitute, in the space in which they exist, the boundaries of the network as components that participate in the realization of the network. ${ }^{14}$

7 Québec, Ministère de la Justice, Rapport de gestion 2009-2010, Québec, 2010, p 37; Jaime Sarophim, « Access Barred: The Effects of the Cuts and Restructuring of Legal Aid in B.C. on Women Attempting to Navigate the Provincial Family Court System » (2010) Rev Can D Fam 26, pp 451-72.

$8 \quad$ LRQ, c C-25, art 61.

9 Rabeea Assy, « Revisiting the Right to Self-representation in Civil Proceedings » (2011) CJQ 30, pp 267-82; Birnbaum et Bala, supra note 4 .

10 Sande L. Buhai, « Access to Justice for Unrepresented Litigants : a comparative Perspective » (2008-09) Loy L Rev 42, pp 979-1020; Camille Cameron et Elsa Kelly, « Litigants in Person in Civil Proceedings : Part 1 » (2002) Hong Kong L J 32, pp 313-42; Lorne D. Bertrand, Joanne J. Paetsch, Nicholas Bala et Rachel Birnbaum, Self-Represented Litigants in Family Law Disputes: Views of Alberta Lawyers, Rapport de recherche déposé au Canadian Research Institute for Law and the Family et à la Fondation du droit de l’Alberta, décembre 2012.

11 Rosemary Hunter, « Adversarial Mythologies: Policy Assumptions and Research Evidence in Family Law » (2003) JL \& Soc’y 30 (1), p 170; Maria De Michele, « La partie qui n’est pas représentée par un avocat », dans Barreau du Québec, Éthique, profession juridique et société, Coll de droit 2011-2012, Vol 13, p 138 [La partie].

12 Tribunal des droits de la personne, Bilan d'activités 2004-2005, Québec, 2005, p 45. Voir également : Québec, Assemblée nationale du Québec, Journal des débats de la Commission des institutions, $37^{\mathrm{e}}$ législature, $1^{\mathrm{re}}$ session, Vol. 38, n 28 (jeudi 29 janvier).

13 Ronald W. Staudt et Paula L. Hannaford, « Access to justice for the self-represented litigant: an interdisciplinary investigation by designers and lawyers » (2002) Syracuse L Rev 52, pp 1017-48; Rory K. Schneider, « Illiberal Construction of Pro Se Pleadings » (2011) U Pa L Rev 159, p 589.

14 Niklas Luhmann, Essay on self-reference, New York, Colombia University Press, 1990, p 3 (entre guillemets dans le texte, citant Humberto Maturana). 
La viabilité du système repose donc en partie sur le processus de sélection des participants, processus qui n’admet que des initiés. C’est du moins ce que nous enseigne la théorie systémique ${ }^{15}$.

Les fondements d'un système de justice contradictoire reposent sur le droit des deux parties de participer au débat et sur leur capacité égale de présenter des arguments ${ }^{16}$. Dans un tel système, les avocats, en tant qu' « officiers de justice " ${ }^{17}$, jouent un double rôle fondamental d'assistance ${ }^{18}$ et de représentation de leur client ainsi que de préparation des dossiers et des débats judiciaires, ce qui assure et permet aux juges une prise de décision impartiale ${ }^{19}$. L'absence des avocats impose donc aux juges, en tant que gardiens de l'équité des procédures, de sortir de leur rôle habituel pour s’assurer que la partie non représentée est bien en mesure de faire valoir ses droits, notamment en l'informant adéquatement ${ }^{20}$. Cet ajustement inévitable du rôle judiciaire remet en question la légitimité des décisions ainsi prises, et donc l'intégrité du système de justice dans son ensemble ${ }^{21}$.

Pour le profane, l’hermétisme du vocabulaire juridique, la complexité des règles procédurales ainsi que le décalage entre la raison juridique - en tant que "vision du monde » - et le sens commun imposent une "frontière » impossible à franchir pour ceux qui ne possèdent pas de " compétences strictement juridiques ${ }^{22}$. Le « recadrage judiciaire ${ }^{23}$ des conflits impose une dépersonnalisation et une distanciation par lesquelles les parties sont dépossédées de leurs histoires et exclues de la recherche de solution ${ }^{24}$.

La constitution d'une compétence proprement juridique, maîtrise technique d'un savoir savant souvent antinomique aux simples recommandations du sens commun, entraîne la disqualification du sens de l'équité des non-spécialistes et la révocation de leur

15 Niklas Luhmann, Systèmes sociaux : esquisse d'une théorie générale, PUL, 2010, et La légitimation par la procédure, PUL, 2001; Gunther Teubner, Droit et réflexivité, Paris et Bruxelles, Coéditions LGDJ, et Bruylant, 1996, et Le droit, un système autopoïétique, PUF, 1993.

16 Anne-Marie Frison-Roche, "La rhétorique juridique », dans Hermès 16, Argumentation et rhétorique (II), éditions CNRS, Paris, 1995, pp 73-83.

17 Talcott Parson les qualifie de « gardiens des institutions légales » : « A Sociologist Looks at the Legal Profession », dans William M. Evan (dir), Law and Sociology, Free Press, New York, 1962, cité dans Cameron et Kelly, supra note 10 à la p 321. Voir également : Andrews c Law Society of British Columbia, [1989] 1 RCS 143, p 187.

18 Le rôle de l'avocat est ainsi d'expliquer le processus légal, de vulgariser le droit et d'offrir un soutien moral, émotif et social : Robert Mnookin et Lewis Kornhauser, « Bargaining in the Shadow of the Law : The Case of Divorce » (1979) Yale L J 88, p 985, repris dans ibid, p 322.

19 Nourit Zimmerman et Tom R. Tyler, « Between Access to Consel and Access to Justice : a Psychological Perspective » (2010) Fordham Urb LJ 37, pp 476-77.

20 Langan, supra note 6 à la p 10. Voir également D. A. Rollie Thompson, « Le juge dans le rôle de conseiller juridique » (printemps 2005) Forum canadien pour la justice civile, pp 3-6 [Le juge].

21 La consultation des décisions de la Cour d'appel du Québec permet de constater que la présence de JNR occasionne régulièrement la remise en question de l’impartialité des juges d’instance.

22 «[L]'institution d'un “espace judiciaire” implique l'imposition d'une frontière entre ceux qui sont préparés à entrer dans le jeu et ceux qui, lorsqu'ils s’y trouvent jetés, en restent en fait exclus, faute de pouvoir opérer la conversion de tout l'espace mental - et en particulier de toute la posture linguistique - que suppose l'entrée dans cet espace social » : Pierre Bourdieu, « La force du droit » (1986) Actes de la recherche en sciences sociales 64, p 9.

23 Pierre Noreau, « La superposition des conflits : limite de l'institution judiciaire comme espace de résolution » (1998) Droit et société 40, p 590.

24 Ibid à la p 609. 
construction spontanée des faits, de leur « vision de l’affaire ». Le décalage entre la vision vulgaire de celui qui va devenir un justiciable, c'est-à-dire un client, et la vision savante de l'expert, juge, avocat, conseiller juridique, etc. n'a rien d'accidentel; il est constitutif d'un rapport de pouvoir, qui fonde deux systèmes différents de présupposés, d'intentions expressives, en un mot, deux visions du monde ${ }^{25}$.

Dans le présent article, nous nous intéresserons à l'affrontement de ces visions antagonistes du monde à la lumière du cas des JNR en matière familiale. La judiciarisation de ces litiges hautement émotifs et de plus en plus complexes ${ }^{26}$, dont les conséquences persistent durant de nombreuses années - à la fois dernier recours et espoir de solution et d'équité - devient parfois le catalyseur, voire l'amplificateur, de tensions déjà existantes ${ }^{27}$. Ce contexte, où les visions du monde et les enjeux ne pourraient être plus opposés, nous semble particulièrement intéressant au regard du discours doctrinal sur les $\mathrm{JNR}^{28}$.

L'hypothèse à la genèse de cette réflexion veut que les interprétations et les stratégies que proposent les juristes s’attachent globalement au maintien de la cohérence générale du système juridique. La vision juridique du monde masquerait ainsi la dimension structurelle de la problématique, qui touche aux prémisses et aux fondements du système, faisant ainsi l'économie des enjeux économiques, politiques et sociaux. Alors que l'accès à la justice apparaît comme une préoccupation constante pour l'ensemble de la communauté juridique, la question épineuse des JNR contribue à mettre en lumière « le caractère traditionnel, archaïque, inadapté des méthodes de travail des tribunaux ${ }^{29}{ }^{29}$

\section{A. Des JNR délibérément perturbateurs}

Les écrits sur la non-représentation sont généralement plus doctrinaux que scientifiques ${ }^{30}$ et visent le plus souvent à proposer des solutions qu'à analyser le problème en tant que question sociale ${ }^{31}$. Ils font

25 Bourdieu, supra note 22 à la p 9.

26 Drew A. Swank, « The Pro Se Phenomenon » (2004) BYU Journal of Public Law 19, pp 373-86.

27 Jessica Pearson, « Court services: Meeting the Needs of Twenty-First Century Families » (1999) Family LQ 33 (3), pp 617-35; Noreau, supra note 23.

28 Nous avons recensé les articles dans les bases de données suivantes : Hein Online, Westlaw, Lexis Nexis, LegalTrac, Legal Periodicals, Azimut, CAIJ, ProQuest et Sage Journals; avec les mots-clés suivants : "pro se litigant », "pro se plaintiff », " unrepresented litigant », " self represented litigant », " litigant in person », " pro se representation », " self representation », « legal representation » \& " without attorney », " legal representation » \& " pro se », « legal representation » \& « litigant ». Des articles trouvés, nous n’avons retenu que ceux dont le sujet est la non-représentation $(\mathrm{N}=268)$. Aux fins de la présente analyse, nous avons sélectionné les articles portant précisément sur les matières familiales ou offrant une vision générale $(\mathrm{N}=81)$. Nous avons procédé à une première analyse inductive à partir d'une sélection aléatoire de dix articles, de laquelle nous avons dégagé de premières pistes d'analyse. Nous avons ensuite commencé l'analyse thématique au moyen du logiciel N’Vivo, enrichissant notre arbre analytique au fur et à mesure.

29 Jacques Commaille et Laurence Dumoulin, « Heurs et malheurs de la légalité dans les sociétés contemporaines. Une sociologie politique de la “judiciarisation” » (2009) L’Année sociologique 59 (1), p 83.

30 Malgré les appels de la communauté scientifique canadienne préconisant le développement de la recherche sur le thème des JNR, particulièrement dans les instances de droit de la famille, les études restent rares. Le sujet commence néanmoins à faire l'objet d'études, notamment des études: Birnbaum et Bala, supra note 4; Macfarlane, The National, supra note 4; Langan, supra note 6.

31 Certains auteurs proposent toutefois une analyse sociologique : Alesha Durfee, « Victim Narratives, Legal Representation, and Domestic Violence Civil Protection Orders » (2009) Feminist Criminology 4 (7), p. 7-31; Macfarlane, ibid; Birnbaum et Bala, ibid 
généralement la description du phénomène en abordant les facteurs explicatifs présumés, les effets sur les instances de divorce ou les processus de médiation ainsi que les stratégies de gestion mises en place par les avocats, les juges et le personnel judiciaire ${ }^{32}$.

Dans le cadre de la présente analyse, nous nous sommes intéressés au discours doctrinal sur les JNR et sur la non-représentation et ses effets. Il émerge de cette analyse trois pistes particulièrement intéressantes : 1- le choix des mots pour nommer les JNR; 2- les JNR comme éléments perturbateurs et perturbés; et 3- la non-représentation en tant que choix délibéré.

\section{Une disparité terminologique}

Nous avons constaté une disparité terminologique parmi les expressions employées pour parler des JNR; le choix des mots est pourtant loin d'être anodin dans ce contexte. De ce que nous avons observé dans la littérature, trois concepts sont fréquemment utilisés. Les termes les plus classiques sont les expressions latines «pro se litigants » ou " pro per litigants » qui signifient «par lui-même » ou " de sa propre personne ». Bien qu'elles soient encore parfois utilisées, elles semblent être progressivement remplacées $^{33}$, principalement par l'expression «self-represented litigants». Quelques auteurs y opposent la notion de "unrepresented litigants » pour mettre en évidence la situation des JNR pour lesquels la non-représentation serait un choix par rapport à ceux pour lesquels il s'agit d'une contrainte $^{34}$. Bien que la plupart des auteurs optent pour l'expression « self-represented » sans expliquer leur choix et sans accorder d'importance aux motifs de la non-représentation, ce choix apparemment anodin semble dénoter un positionnement non dévoilé. Ainsi, le "self-represented litigant » apparaît comme un élément perturbateur, un problème à régler, alors que le "unrepresented litigant » serait plutôt une victime à protéger.

\section{Les JNR comme éléments perturbateurs et perturbés}

Les JNR sont généralement dépeints comme des éléments déstabilisants et perturbateurs; leur présence constituerait une «crise », un «fardeau », voire un «cauchemar » ${ }^{35}$. Pour la majorité des

32 Par ex. : Bruce D. Sales, Connie J. Beck et Richard K Hann, « Is Self-Representation a Reasonable Alternative to Attorney Representation in Divorce Cases » (1992) ST Louis U LJ 37, pp 553-606; Carolyn D. Schwarz, « Pro Se Divorce Litigants - Frustrating the Traditional Role of the Trial Court Judge and Court Personnel » (2004) FCR 42 (4), pp 655-72; Jona Goldschmidt, « Judicial Assistance to Self-Represented Litigants: Lessons from the Canadian Experience » (2008) Mich St J Int’l L 17, pp 601-56; Rosselle L. Wissler, « Representation in Mediation: what we know from Empirical Research » (2010) Fordham Urb LJ 37, pp 419-71; Michael M. Pettersen, Robin H. Ballard, John W. Putz et Amy Holtzworth-Munroe, « Representation disparities and impartiality: An empirical analysis of party perception of fear, preparation, and satisfaction in divorce mediation when only one party has counsel » (2010) FCR 48 (4), pp 663-71; Pearson, supra note 27.

33 Comme c'est le cas de l'ensemble des expressions latines : Berenson, supra note 5 à la p 107.

34 Berenson, supra note 5 à la p 107; Russell Englers, « And Justice for All - Including the Unrepresented Poor: Revisiting the Role of the Judges, Mediators and Clercks » (1987) Fordham L Rev 67, p 1988; Langan, supra note 6, para 2; Richardson, Sourdin et Wallace, supra note 5 aux pp 4-12. En français, un seul article établit la même distinction entre les expressions « plaideurs qui se représentent eux-mêmes » et « plaideurs non représentés »: Thompson, Le juge, supra note 20 à la p 3.

35 Englers, supra note 34 à la p 1987; Cameron et Kelly, supra note 10 à la p 316; Schneider, supra note 13 à la p 597 ; Melinda Buckley, The Legal Aid Crisis: Time for Action, Association du Barreau Canadien, Ottawa, 2000, p 45; Berenson, supra note 5 à la p 105; Karl Branting, « An Advisory System for Pro Se Protection Order Applicants » 
auteurs, le problème serait l'incapacité des JNR à se conformer aux multiples exigences du processus judiciaire $^{36}$. La première difficulté résiderait dans la rigidité du cadre procédural ${ }^{37}$ : la nature contradictoire du débat judiciaire, qui relègue aux parties plutôt qu'au juge la gestion et le déroulement de l'instance $^{38}$, requiert la maîtrise de connaissances et d'habiletés développées habituellement à la suite d'une formation échelonnée sur plusieurs années. Les éléments procéduraux qui constituent les principales embûches sont : les règles complexes d'admissibilité de la preuve, l'usage d'expertises, la désignation de la partie opposée, la prescription et les délais, les exigences de forme pour les procédures écrites, le fonctionnement des mécanismes de médiation, les changements inopinés de juge ${ }^{39}$, etc. Dans la même veine, la deuxième difficulté est relative à la croissance d'un corpus législatif de plus en plus complexe dont les JNR ne pourront pas nécessairement saisir toutes les subtilités ${ }^{40}$. Les incidences d'une méconnaissance des règles procédurales et substantielles peuvent être lourdes de conséquences sur le cours de l'instance ${ }^{41}$ - les juges retenant généralement les arguments de la partie représentée, conformes au droit applicable ${ }^{42}$ - et générer un sentiment d'injustice, particulièrement dans les cas où les JNR font face à des parties représentées ${ }^{43}$.

La troisième difficulté, identifiée par une minorité d'auteurs, est constituée des barrières culturelles et cognitives entre le système juridique et le monde ordinaire : le mode de raisonnement et les techniques discursives inhérents au système juridique diffèrent en effet grandement des pratiques populaires. La

(2000) IRLCT 14 (3), p 357; Jona Goldschmidt, Barry Mahoney, Harvey Solomon et Joan Green, Meeting the Challenge of Pro Se Litigation: A Report and Guidebook for Judges and Court Managers, American Judicature Society, Chicago, 1998, pp 52-54; Richard W. Painter, « Pro Se Litigation in Times of Financial Hardship - A Legal Crisis and Its Solutions » (2011) Family L Q 45 (1), pp 45-94; Deborah Doherty, « Promoting Access to Family Justice by Educating the Self-Representing Litigants » (2012) RD UN-B 63, p 86.

Paula Hannaford-Agor, The Future of Self-Represented Litigation: Report From the March 2005 Summit, National Center for State Courts, 2005, pp 163-64; Richardson, Sourdin et Wallace, supra note 5 à la p 14; Schneider, ibid à la p 598.

37 Cameron et Kelly, supra note 10 aux pp 318, 323 et 336; Connie J. A. Beck, Michele E. Walsh, Robin H. Ballard, Amy Holtzworth-Munroe, Amy G. Applegate et John W. Putz, « Divorce Mediation with and without Legal Representation: a Focus on Intimate Partner Violence and Abuse » (2010) FCR 48 (4), p 632; Berenson, supra note 5 à la p 115; Branting, supra note 35 à la p 358; Buhai, supra note 10 aux pp 984-86; Langan, supra note 6, para 38; Macfarlane, The National, supra note 4 aux pp 95 et ss.

38 Art 4.1 Cpc; Richard Moorhead, « The Passive Arbiter: Litigants in Person and the Challenge To Neutrality » (2007) Social \& Legal Studies 16 (3), pp 405-24; Englers, supra note 34; Langan, supra note 6.

39 Moorhead, ibid à la p 409; Assy, supra note 9 à la p 25; Beck, Walsh, Ballard, Holtzworth-Munroe, Applegate et Putz, supra note 37 à la p 632; Cameron et Kelly, supra note 10 à la p 323; Durfee, supra note 31 aux pp 23-27; Langan, supra note 6, para 15; Macfarlane, The National, supra note 4 à la p 95; Pettersen, Ballard, Putz et Holtzworth-Munroe, supra note 32 à la p 669.

40 Richard Zorza, « An Overview of Self-Represented litigation Innovation, Its Impact, and Approach to the Future: An Invitation to Dialogue » (2009) Fam L Q 3 (3), p. 535 [An Overview]; Charles R. Dyer, « The Queen of Chula Vista: Stories of Self-Represented Litigants and a Call for Using Cognitive Linguistics to Work with Them » (2007) Law Lib J 99 (4), p 727; Branting, supra note 35 à la p 558; Buhai, supra note 10 à la p 984; Cameron et Kelly, supra note 10 à la p 324; Pettersen, Ballard, Putz et Holtzworth-Munroe, ibid à la p 669.

41 Birnbaum et Bala, supra note 4 à la p 113; Buhai, supra note 10 à la p 986. Voir également Rebecca Sandefur, « Effects of Representation on Trial and Hearing Outcomes in Two Common Law Countries », conférence présentée au colloque de l'Association internationale de sociologie, Paris, 12 juillet 2005, p 24.

42 Berenson, supra note 4 à la p 115; Moorhead, supra note 38 aux pp 409-10.

43 Moorhead, ibid à la p 414; Durfee, supra note 31 à la p 10. 
structure thématique du récit, la sélection des personnes et des évènements ainsi que leur description, l'ordre de présentation des éléments factuels, le langage utilisé et sa signification sont autant d'éléments distinctifs du champ juridique ${ }^{44}$.

Self-represented litigants must learn the meanings behind the legal discourse, learn how to apply legal discourse properly, and learn to accept the analysis of the situation that the discourse would give it. It is somewhat like learning a whole new culture. ${ }^{45}$

Les récits élaborés par des avocats, ou avec leur assistance, sont ainsi généralement jugés plus crédibles et offrent de meilleures chances d'obtenir gain de cause ${ }^{46}$.

Pour certains auteurs néanmoins, les prémisses du système juridique sont au cœur du problème que met en exergue le phénomène de la non-représentation ${ }^{47}$. Ces prémisses seraient illusoires, voire erronées. L’égalité formelle des parties, la logique purement contradictoire, l'interprétation du devoir d'apparence d'impartialité des juges, les rôles fixes et prédéterminés des acteurs juridiques, etc. ne constitueraient qu’une rhétorique décalée des réalités sociales et juridiques. Dans cette perspective, seule une restructuration du système et de la raison juridiques pourrait réellement permettre l'inclusion des $\mathrm{JNR}^{48}$.

\section{Le choix délibéré de la non-représentation}

Le phénomène de la non-représentation est souvent dépeint comme une problématique particulièrement complexe $^{49}$, liée à l'évolution sociale et juridique ${ }^{50}$. Cependant, en raison du peu de recherches en la matière, les analyses rigoureuses sur la question sont rares, et les écrits, souvent fondés sur des observations et des anecdotes, ne font généralement pas de place aux effets du contexte social, notamment économique.

Ainsi, pour plusieurs auteurs, le fait de ne pas être représenté est d'abord le résultat d'un choix délibéré $^{51}$. La majorité des JNR seraient des «individus qui ont perdu confiance envers les avocats et

44 Durfee, ibid aux pp 10-14; Dyer, supra note 40 aux pp 728-729, 731.

45 Dyer, ibid à la p 731.

46 Durfee, supra note 31 aux pp 57-58.

47 Par ex. : Zorza, An Overview, supra note 40 aux pp 519-44; Englers, supra note 34; Russell G. Pearce, « Redressing Inequality in the Market for Justice: Why Access to Lawyers Will Never Solve the Problem and Why Rethinking the Role of Judges Will Help » (2004) Fordham L Rev 73, pp 969-80; Macfarlane, The National, supra note 4.

48 Englers, supra note 34 aux pp 2022-24, 2028 et ss; Richard Zorza, « The Disconnect Between the Requirements of Judicial Neutrality and Those of the Appearance of Neutrality when Parties Appear Pro Se: Causes, Solutions, Recommendations, and Implications » (2003-2004) Geo. J. Legal Ethics 17, pp 423-54 [The Disconnect].

49 Rachel Birnbaum, Nicholas Bala et Lorne Bertrand, « The Rise of Self Representation in Canada’s Family Court: the Complex Picture Revealed in Surveys of Jugdes, Lawyers and Litigants » (2012) R du B Can 91, p 94; Birnbaum et Bala, supra note 4 à la p 104.

50 Par ex. : l'étude de l’évolution de la pratique du divorce montre une corrélation entre l'accessibilité au divorce et l'accroissement des instances et des JNR : Judith G. McMullen et Debra Oswald, « Why Do We Need a Lawyer?: An Empirical Study of Divorce Cases » (2010) JL \& Fam Stud 12, p 63.

51 Par ex : Tétrault, supra note 4 à la p 205; Cameron et Kelly, supra note 10 à la p 318. 
[...] croient sincèrement pouvoir faire un meilleur travail à défendre leurs propres intérêts » ${ }^{52} ; 73$ pour cent des avocats albertains partagent cette opinion ${ }^{53}$. La culture "do-it yourself » est ainsi régulièrement invoquée pour expliquer le choix de ne pas être représenté ${ }^{54}$. La difficulté de saisir la complexité des enjeux juridiques et du processus judiciaire donnerait à certains une impression de simplicitéé ${ }^{55}$, renforçant paradoxalement la conviction de pouvoir facilement passer au travers du processus ${ }^{56}$. La « prolifération » de la vente de formulaires vulgarisés, de trousses d’information juridique et de services d'assistance ainsi que l'accès facilité à l'internet conforteraient les JNR dans leur impression de capacité et d'autonomie ${ }^{57}$. En même temps, certains JNR voudraient par leur présence forcer le tribunal à envisager leur problème de la même manière qu'eux, et non en fonction de critères juridiques ${ }^{58}$.

Le choix de l'autoreprésentation serait parfois le résultat d'un calcul coût/bénéfice. Dans certains cas, les JNR disposeraient des moyens financiers de se procurer une représentation, mais ne seraient pas convaincus de la réelle valeur du service ${ }^{59}$. Dans d'autres cas, les JNR voudraient assumer le plein contrôle de leur litige; l'avocat constituerait alors un frein à leurs objectifs personnels ${ }^{60}$. Dans d'autres cas encore, les JNR opteraient pour la non-représentation pensant obtenir la sympathie du juge et ainsi gagner leur cause ${ }^{61}$.

La non-représentation serait parfois le résultat d'expériences malheureuses avec des avocats - la difficulté d'en trouver un, l'impression que l'avocat n'a fait rien dans le dossier, qu'il n'est pas à l'écoute, ne donne pas suffisamment de renseignements, ne fait pas d'efforts pour parvenir à un arrangement à l'amiable ou n'est tout simplement pas compétent - ou encore d'une décision judiciaire défavorable ${ }^{62}$.

Bien que le choix délibéré de la non-représentation soit fréquemment invoqué ${ }^{3}$, des études confirment depuis plusieurs années que ce sont d’abord pour des raisons de nature financière que les

52 De Michele, Seul, supra note 3 à la p 5. Cette tendance aurait pour origine la « désintermédiation », par laquelle l’individu délègue de moins en moins des actes qui jusqu'alors était l'apanage exclusif de professionnels : Berenson, supra note 5 à la p 121.

53 Bertrand, Paetsch, Bala et Birnbaum, supra note 10 à la p 4.

54 Berenson, supra note 5 à la p 119. Voir également : Sales, Beck et Hann, supra note 32 à la p 598; Beck, Walsh, Ballard, Holtzworth-Munroe, Applegate et Putz, supra note 37 à la p 632; Richardson, Sourdin et Wallace, supra note 5 à la p 17; Macfarlane, The National, supra note 4 à la p 35; Birnbaum, Bala et Bertrand, supra note 49 aux pp 71, 74-78, 93.

55 Berenson, ibid à la p 120; Cameron et Kelly, supra note 10 à la p 319; Langan, supra note 6, para 25; McMullen et Oswald, supra note 50 aux pp 57-59; Robert B. Yegge, « Divorce Litigants without Lawyers » (1994) Fam L Q 28 (3), pp 410-11.

56 Cette impression de simplicité ne serait cependant pas la même selon les domaines (Berenson, ibid à la p 119) et la qualité des parties, les demandeurs étant plus souvent représentés que les défendeurs (Zimmerman et Tyler, supra note 19 à la p 493).

57 Berenson, ibid à la p 120.

58 Zimmerman et Tyler, supra note 19 à la p 500.

59 Richardson, Sourdin et Wallace, supra note 5 à la p 16; Birnbaum, Bala et Bertrand, supra note 49 aux pp 71 et 76 ; Macfarlane, The National, supra note 4.

60 Jennifer Blishen, « Self-Represented Litigants in Family and Civil Law Disputes » (2006) Can Fam Law Q 25 (2), pp 117-18.

61 Blishen, ibid à la p 118.

62 Kim Williams, Litigants in person: a literature review, Ministry of Justice, United Kingdom, 2011, p 4-5; Macfarlane, The National, supra note 4 aux pp 44-48.

63 Swank, supra note 26 aux pp 378-79. 
justiciables se présentent seuls devant les tribunaux ${ }^{64}$. Une étude de terrain a démontré que seulement 20 pour cent des JNR ont choisi de ne pas payer pour les services d'un avocat du fait qu'ils préféraient prendre en charge leur dossier eux-mêmes, alors que 90 pour cent d'entre eux évoquaient des raisons pécuniaires pour expliquer leur non-représentation ${ }^{65}$. Plus de la moitié des répondants avaient eu recours aux services d'un avocat, mais n'étaient plus capables de payer ${ }^{66}$, et 86 pour cent d'entre eux ont déclaré avoir cherché, sans succès, une représentation juridique à un prix accessible. ${ }^{67}$

Les honoraires des avocats et les frais judiciaires seraient donc directement à l'origine du phénomène d'accroissement des JNR dans les instances judiciaires ${ }^{68}$. Ces dernières décennies, en raison du prix élevé des services juridiques - qui serait déterminé par la richesse des clients commerciaux en mesure de se les payer ${ }^{69}$ - et de l'altération des conditions d'admissibilité à l'aide juridique, des pans entiers de la population sont devenus incapables de se payer un avocat sans pour autant être admissibles à l'aide juridique $^{70}$. Une proportion importante de juges américains aurait d'ailleurs remarqué une recrudescence de la présence de JNR depuis la crise financière de $2008^{71}$.

\section{B. Des palliatifs et des expédients}

Alors que l'efficacité et la fonctionnalité du système contradictoire reposent sur le droit des parties de participer au débat judiciaire, plusieurs auteurs considèrent logiquement la promotion et l'accroissement

64 Macfarlane, The National, supra note 4; Birnbaum, Bala et Bertrand, supra note 49; Richardson, Sourdin et Wallace, supra note 5 à la p 14; Robert B. Yegge, « Divorce Litigants Without Lawyers: This crisis for bench and bar needs answers now » (1994) Judges J 33, p 12. Voir également : Beverley McLachlin, « Reflections - The Challenges We Face » (2007) UBCL Rev 40, p. 819-28 aux paras 7-14. En matière de divorce, le revenu du marié serait le meilleur indicateur de non-représentation : McMullen et Oswald, supra note 50.

65 Macfarlane, ibid aux pp 39 et 48. Voir également : Langan, supra note 6, para 14. Certaines recherches aboutissent cependant à des résultats mitigés : dans l'étude de Sales, Beck et Hann (supra note 32 à la p 567), les raisons invoquées par les JNR pour expliquer la non-représentation est la simplicité des procédures (45 pour cent), puis l’incapacité de payer les honoraires d'un avocat (31 pour cent), alors que dans celle de Birnbaum, Bala et Bertrand (supra note 49 à la p 78), 90 pour cent des juges et 45 pour cent des JNR ont affirmé que la principale raison était d'ordre économique.

66 Macfarlane, ibid à la p 42.78 pour cent des québécois considèrent ne pas avoir les moyens de se présenter devant les tribunaux : Lafond, supra note 6, p. 29.

67 Blogue de Julie Macfarlane, 3 assumptions that are leading us astray - and impeding our commitment to system reform that could enhance access to justice, http://drjuliemacfarlane.wordpress.com/2013/10/28/3-assumptions-that-are-leadingus-astray-and-impeding-our-commitment-to-system-reform-that-could-enhance-access-to-justice/ (consulté le 28 octobre 2013).

68 Macfarlane, The National, supra note 4 à la p 40; Berenson, supra note 5 à la p 117; Comité d'action sur l'accès à la justice en matière civile et familiale, L'accès à la justice en matière civile et familiale - Une feuille de route pour le changement, Ottawa, 2013, p 5.

69 Lafond, supra note 6 à la p 57.

70 Sarophim, supra note 7; Cameron et Kelly, supra note 10 à la p 328; Jonh Dewar, Barry W Smith et Cate Banks, Litigants in Person in the Family Court of Australia, rapport de recherche déposé à la Family Court of Australia, 2000, pp 11-12; Blishen, supra note 60 à la p 117. Pour Richardson, Sourdin et Wallace, le lien entre les restrictions budgétaires à l’aide juridique et la hausse des JNR n’est pas clairement établi : supra note 5 à la p 16.

71 Richard Zorza, « Access to Justice: Economic Crisis Challenges, Impacts, and Responses », dans Carol L. Flango, Amy M. McDowell, Charles F. Campbell et Neal B. Kauder (dir.), Future Trends in State Courts 2009, National Center for State Courts, Williamsburg 2009, pp 9-12. Voir également Painter, supra note 35. 
de la représentation par avocat comme les solutions privilégiées ${ }^{72}$. Les effets perturbateurs de la nonreprésentation, à la fois sur les droits des JNR et sur l'efficacité et la légitimité du système judiciaire, sont souvent posés en termes d'accès à la justice ${ }^{73}$. Ces auteurs proposent notamment : l'investissement massif de l'État pour favoriser l'accessibilité à la représentation ${ }^{74}$, y compris par le rehaussement des plafonds d'admissibilité à l'aide juridique ${ }^{75}$, le développement des services pro bono ${ }^{76}$, la mise en place d'assurances juridiques ${ }^{77}$, la réduction des honoraires des avocats ${ }^{78}$, la possibilité pour les tribunaux civils d'ordonner la représentation dans les cas où son absence crée un déséquilibre ${ }^{79}$ ainsi que la reconnaissance d'un droit civil ou fondamental à la représentation par avocat ${ }^{80}$.

Outre l'universalité de la représentation, les solutions proposées dans les cas où un JNR se présente au tribunal peuvent être classées sous trois catégories : 1- le renforcement des compétences et des aptitudes juridiques des JNR; 2- la formation et la préparation des différents protagonistes du système juridique pour qu'ils soient en mesure de faire face à la situation; et 3- les aménagements internes au système, notamment la simplification de la procédure et l'utilisation de moyens alternatifs de résolution des conflits [MARC].

\section{Le renforcement des compétences et des aptitudes juridiques des JNR}

Après la promotion de la représentation par avocat, il s'agit de la catégorie de solutions de loin la plus abondante dans la doctrine. Elle est souvent présentée comme un moyen d'autonomisation («empowerment») pour les JNR, une façon de reprendre une forme de contrôle sur le processus judiciaire, voire de bonifier un statut citoyen largement fondé sur la revendication et l'exercice des droits $^{81}$. Des JNR mieux outillés sont aussi moins perturbateurs pour les acteurs judiciaires et le fonctionnement du système dans son ensemble.

72 Tous ne partagent cependant pas cette opinion. Par ex., pour Pearce, les juges doivent être responsabilisés au regard des inégalités persistant en matière d'accès à la justice : supra note 47.

73 Par ex. : Buhai, supra note 10; Gord MacDonald et Helena Birt, « Duty Counsel and the Self-represented Litigant » (2001) Can Fam Law Q 19, pp 497-507; Linda F. Smith et Barry Stratford, « DIY in Family Law: a Case Study of a brief Advice Clinic for Pro Se Litigants » (2012) JL \& Fam Stud 14, p 215; De Michele, La partie, supra note 11.

74 Deborah J. Cantrell, « Justice for Interest of the Poors: the Problem of Navigating the System without Counsel » (2002) Fordham L R 70, p 1575.

75 Julie MacFarlane, Opening the Dialogue: The SRL Phenomenon, conférence donnée à la Faculté de droit de l’Université de Windsor, Opening the Dialogue: The SRL Phenomenon, 9 au 11 mai 2013 [en ligne : http://representingyourself.com/dialogueevent.html] (consulté le 26 octobre 2013) [Opening]; Langan, supra note 6, paras 19 et ss.

76 Berenson, supra note 5 aux pp 133 et ss; Langan, supra note 6, para 57; Leslie Feitz, « Pro Se Litigants in Domestic Relation Cases » (2008) JAAML 21, p. 201; Jim Hilbert, « Educational Workshops on Settlement and Dispute Resolution: Another Tool for Self-Represented Litigants in Family Court » (2009) Family L Q 43 (3), p 556.

77 Buhai, supra note 10 à la p 1009.

78 Berenson, supra note 5 aux pp 130 et ss.

79 Assy, supra note 9; Schwarz, supra note 32.

80 Earl Johnson Jr., « Will Gideon's Trumpet Sound a New Melody? The Globalization of Constitutional Values and Its Implications for a Right to Equal Justice in Civil Cases » (2003) SEAJSJ 2, pp 201-31; Buhai, supra note 10 à la p 1011.

81 MacFarlane, Opening, supra note 75; Elizabeth McCulloch, « Let Me Show How: Pro Se Divorce Courses and Client Power » (1996) Fla L Rev 48, pp 481-508. 
Il s’agit ici essentiellement d'assurer un meilleur accès à l'information juridique en aménageant des sites internet, des lignes téléphoniques ou des sessions d'information ${ }^{82}$, en simplifiant, en vulgarisant et en traduisant les contenus ${ }^{83}$, et en proposant de nouveaux services, notamment dans les bibliothèques de droit ou encore directement dans les tribunaux ${ }^{84}$. En plus de l'offre de formulaires et de dépliants qui présentent les différentes étapes du processus judiciaire ${ }^{85}$, ces services peuvent prendre la forme de sessions obligatoires d’information, de kiosques animés par des bénévoles ${ }^{86}$, de la présence d'avocats de garde $^{87}$, de centres d'éducation et d'information ( «Self-Help Center ») ${ }^{88}$ ou de cliniques destinées à aider les JNR («Pro Se Clinics ») ${ }^{89}$. Alors qu'une partie de l'information juridique est offerte par des canaux officiels, certaines initiatives sont le fait d'organisations privées, d'organismes communautaires et d'OSBL; cette disparité d'offres peu encadrées fait craindre une inégalité de la qualité des services et une hausse incontrôlée des frais ${ }^{90}$.

En effet, la connaissance et la maîtrise de notions complexes de droit procédural et substantiel demandent plus que de la simple information : il s'agit d'assister, voire de « coacher » les JNR, nonseulement dans la préparation des dossiers, mais également pour les interrogatoires, les plaidoiries, etc ${ }^{91}$. Le « coaching » doit en définitive accorder une certaine autonomie décisionnelle aux JNR ${ }^{92}$. Dans certains bureaux d'avocats, les justiciables peuvent obtenir des avis juridiques, notamment au moyen de services en ligne, et parfois même une aide à la préparation du dossier qu'ils iront ensuite présenter euxmêmes $^{93}$ : c’est le mandat à portée limitée («Unbundled Legal Services ») ${ }^{94}$. Le « coaching » peut

82 Par ex. : L'informelle - Une référence en droit familial, qui propose une ligne téléphonique d'information juridique gratuite et des séances d’information juridique, [en ligne : www.informelle.osbl.ca/] (consulté le 5 septembre 2013).

83 Voir la série de fascicules « Seul devant la Cour » proposée par la Fondation du Barreau du Québec, [en ligne : www.fondationdubarreau.qc.ca/publications/seul/] (consulté le 6 septembre 2013). Julie MacFarlane propose par exemple que des résumés jurisprudentiels soient rendus disponibles : Opening, supra note 75. Voir également : Chase, supra note 4 à la p 416.

84 Cameron et Kelly, supra note 10 à la p 324; Margaret B. Flaherty, « How Courts Help to Help Yourself: The Internet ans the Pro Se Divorce Litigant » (2002) FCR 40 (1), pp 91-115.

85 Cette stratégie permet de pallier le manque de disponibilité du personnel judiciaire : Schwarz, supra note 32 à la p 665. Voir par ex la section Ressources pour les plaideurs non représentés du site de la Cour suprême du Canada : www.scccsc.gc.ca/res/unrep-nonrep/index-fra.aspx] (consulté le 5 septembre 2013) et les aide-mémoire disponibles sur les sites de la Cour d’appel et de la Cour supérieure du Québec : <www.tribunaux.qc.ca/c-appel/Faq/memoire_faq.html> et $<$ www.tribunaux.qc.ca/c-superieure/indexcs.html> (consultés le 5 septembre 2013).

86 Zorza, An Overview, supra note 40 à la p 522.

87 En Ontario, des centres d’information juridique, où œuvrent des avocats payés par l'aide juridique, sont aménagés directement dans les palais de justice. Ces avocats peuvent rencontrer tout justiciable durant 20 minutes, mais ne peuvent offrir leurs services qu'aux personnes se qualifiant selon les critères de l'Aide juridique : Langan, supra note 6. Au Québec, le Barreau de Montréal a mis sur pied un service de garde visant à offrir une aide ponctuelle en matière familiale à toute personne dont la cause est inscrite au rôle de la chambre de pratique : [en ligne : www.barreaudemontreal.qc.ca/pages/FP102b_servgarde.html] (consulté le 6 septembre 2013).

88 Hilbert, supra note 76 à la p 555.

89 Berenson, supra note 5 à la p 122; Chase, supra note 4 aux pp 417 et ss; Smith et Stratford, supra note 109.

$90 \quad$ Ibid à la p 128.

91 Cameron et Kelly, supra note 10 à la p 324.

92 Certains vont jusqu'à parler de «mentorat » («mentoring ») : Macfarlane, The National, supra note 4 à la p 80.

93 Par ex. : Autoreprésentation.com - Services juridiques à bas prix, où l’on peut avoir accès à de l'information juridique ou à des avis juridiques ainsi qu'à un soutien pour faire des recherches sur les lois et la jurisprudence. Un avocat peut même rédiger une partie des documents juridiques. Tout se passe virtuellement : <www.autorepresentation.com> (consulté le 5 
cependant aller encore plus loin et faire appel à des professionnels de la communication, de la négociation ou de la représentation. Les outils à développer ne sont, dans ce cas, plus seulement ceux du droit, mais plutôt ceux de la socialisation dans le contexte judiciaire ${ }^{95}$.

En plus du "coaching ", par lequel on vise à développer des compétences, plusieurs auteurs soulignent l'importance du soutien psychologique et moral des JNR. Ce soutien peut prendre plusieurs formes: l'intervention professionnelle de travailleurs sociaux ou de psychologues, la constitution d'ateliers sur le fonctionnement du système judiciaire et sur les effets des décisions de justice ainsi que la présence réconfortante des proches ${ }^{96}$.

L'ensemble de ces solutions demande parfois l'intervention de non-juristes dont le rôle est le plus souvent d'informer et d'accompagner. Alors que certains valorisent l'intégration des services juridiques, sociaux et cliniques ${ }^{97}$, d'autres suggèrent de reconnaître officiellement la compétence de non-juristes pour informer et assister les $\mathrm{JNR}^{98}$. Cette proposition est hautement controversée ${ }^{99}$, puisque seuls des membres du Barreau peuvent donner des conseils juridiques et assurer une représentation. Pour Julie MacFarlane, il revient au politique de faire l'évaluation des besoins des JNR et de l'offre de services parajuridiques, en tenant compte des situations juridiques requérant nécessairement l'intervention d'un avocat $^{100}$.

Une bonne qualité d'information et un encadrement efficace des JNR constituent, du point de vue de certains auteurs, le meilleur moyen de libérer les juges et le personnel judiciaire du fardeau d'une prise en charge complexe et coûteuse ${ }^{101}$. Plusieurs soulignent néanmoins l'échec des mesures mises en place jusqu'à maintenant pour assurer aux JNR, surtout dans les cas complexes et difficiles, une qualité d'information et de représentation adéquate ${ }^{102}$.

Si le renforcement des compétences et des aptitudes juridiques des JNR est une solution peu coûteuse et facile à mettre en œuvre, les retombées positives ne sont pas clairement établies ${ }^{103}$. Il semblerait que ces modes d'information et de conseil ne permettent pas, dans plusieurs des cas, de remplacer efficacement l'analyse et l'argumentaire juridique d'un avocat ${ }^{104}$, et des études démontrent qu'ils n'ont

septembre 2013); Avocat à la carte - Services juridiques sur mesure, où l'on propose «l'aide d'un avocat pour faire toutes les procédures judiciaires (mise en demeure, requête, réponse, interrogatoire, opinion juridique, préparation de l'audition, etc.) » : <www.avocatalacarte.com> (consulté le 5 septembre 2013).

Langan, supra note 6, paras 55-56; Feitz, supra note 76 à la p 202; Hilbert, supra note 76 à la p 556. Voir à ce sujet :

Barreau de Montréal, Mandats à portée limitée : guide à l'intention des avocats, Montréal, 2011 [en ligne :

www.barreaudemontreal.qc.ca/loads/Guides/GuideMandatPorteeLimitee_fr.pdf] (consulté le 7 septembre 2013). Macfarlane, The National, supra note 4 à la p 120.

96 MacFarlane, Opening, supra note 75; Hilbert, supra note 76 aux pp 558 et ss; Cameron et Kelly, supra note 10 à la p 325; Macfarlane, The National, supra note 4 à la p 77.

97 MacFarlane, Opening, supra note 75.

98 Langan, supra note 6, para 54. Voir également: Fortin c Chrétien, [2001] 2 RCS 500.

99 Berenson, supra note 5 à la p 128.

100 Macfarlane, The National, supra note 4 aux pp 193 et ss.

101 Schwarz, supra note 32; Feitz, supra note 76 à la p 202.

102 Lynn Mather, « Changing Patterns of Legal Representation in Divorce: From Lawyers to Pro Se » (2003) JL \& Soc'y 30, p 155.

103 Buhai, supra note 10 à la p 993.

104 Birnbaum, Bala et Bertrand, supra note 49 à la p 93; Richard Zorza, The Self-Help Friendly Court: Designed from the Ground Up to Work for People Without Lawyers, The National Center for State Courts, Williamsburg, 2002, pp 17 et ss [The Self-Help]. Soulignons que les différentes solutions proposées en matière d'information supposent une capacité 
pas d’incidence sur les perspectives de gain pour les JNR en ayant bénéficié comparativement à ceux qui n’y ont pas eu accès ${ }^{105}$.

\section{La formation et la préparation des protagonistes du système juridique}

L’information et l'assistance proposées aux JNR sont offertes en partie par le personnel judiciaire. Ce personnel n'est pas habilité à donner des avis juridiques ${ }^{106}$. Toutefois, l'augmentation du nombre de justiciables ayant besoin d'aide pratique et de renseignements aurait entraîné le développement d'une pratique informelle d'information ${ }^{107}$. Cependant, en raison du flou entre renseignements et conseils juridiques ainsi que des conséquences d'un éventuel exercice illégal du droit, le personnel judiciaire resterait prudent, laissant parfois des justiciables désemparés sans réponse ${ }^{108}$. Une étude menée dans quelques provinces canadiennes démontre que le personnel judiciaire, bénéficiant de peu de formation, serait mal préparé pour répondre aux demandes de justiciables confus et anxieux. Frustré de ne pas être en mesure d'assister convenablement les JNR, il subirait un stress professionnel important ${ }^{109}$.

Il apparaît assez clairement que les efforts en termes de préparation et de formation se concentrent davantage sur les juristes actifs dans l'arène judiciaire, et plus particulièrement sur les avocats et les juges. La présence régulière de JNR est l'objet de la préoccupation croissante des associations juridiques, professionnelles ou non. Depuis quelques années, les publications et les prises de position sur le sujet se multiplient ${ }^{110}$, de même que les articles et les conférences de juges ${ }^{111}$ et d'avocats ${ }^{112}$. Des ateliers et formations sur le sujet se donnent régulièrement ${ }^{113}$.

d'accès physique (accès à internet, à une imprimante, etc.) et cognitif (capacité de lecture notamment).

105 Buhai, supra note 10 à la p 993. Voir également Gary Blasi, «Framing Access to Justice: Beyond Perceived Justice for Individuals », (2009) 42 Loy L A L Rev 913, pp 919-20; Gary Blasi, « How Much Access? How Much Justice? », (2004) 73 Fordham L Rev 865, pp 869-70; Carroll Seron, Martin Frankel, Gregg Van Ryzin et Jean Kovath, « The Impact of Legal Counsel on Outcomes for Poor Tenants in New York City's Housing Court: Result of a Randomized Experiment » (2001) L \& Soc'y Rev 35, p 419.

106 D’après un dépliant de la Fondation du Barreau du Québec, le personnel du greffe est autorisé à donner des renseignements sur les types de formulaires, la façon de les remplir et les tarifs, à diriger le public vers les ressources adéquates et à expliquer certaines notions procédurales : Seul devant la Cour en matière familiale, Fondation du Barreau du Québec, [en ligne :

www.fondationdubarreau.qc.ca/pdf/publication/seul-devant-la-cour-familiale-fr.pdf], p 13 (consulté le 6 septembre 2013).

107 Berenson, supra note 5 à la p 125; Macfarlane, The National, supra note 4 aux pp 67 et ss.

108 Schwarz, supra note 32 aux pp 663 et ss; Chase, supra note 4 à la p 423; Macfarlane, ibid à la p 117.

109 Macfarlane, The National, supra note 4 aux pp 62-63, 68 et 118. Voir également Schwarz, supra note 32 à la p 664; Donna Stienstra, Jared Bataillon et Jason A. Cantone, Assistance to Pro Se Litigants in U.S. District Courts: A Report on Surveys of Clerks of Court and Chief Judges, Federal Judicial Center, Washington, 2011, p 13.

110 Par ex. : Conseil canadien de la magistrature, Énoncé de principes concernant les plaideurs et les accusés non représentés par un avocat, Ottawa, 2006; Association du Barreau canadien, Une cause justifiée - Le droit à une représentation juridique rémunérée par l'État au Canada, Ottawa, 2002; Fondation du Barreau du Québec, Possible et actuelle : une plus grande humanisation du droit de la famille ?, Québec, 1997; Forum canadien sur la justice civile, « Accès aux services juridiques » (2009), Idées et actualités sur la réforme de la justice civile 12.

111 Association canadienne des juges des cours provinciales, " Accès à la justice et plaideur non représenté » (2009-10) Journal des juges provinciaux 32 (2); Forum des juges de l'Association du Barreau canadien, « Les justiciables non représentés par avocat » Vox Judicia, mai 2000 ; Beverley McLachlin, supra note 66 aux 819-28; Élizabeth Corte, Une juge en chef raconte, déjeuner-causerie, Québec, 20 avril 2011; Michel Robert, La magistrature à l'ère du jugement sur mesure, colloque éducaloi : Dire le droit pour être compris, octobre 2010. 
Le défi majeur, pour les avocats comme pour les juges, est celui de l'humanisation d'un système de justice complexe et inaccessible à plusieurs égards, tout en préservant les conditions de fonctionnement qui permettent la mise en œuvre des droits et l'adjudication. Ainsi, même si le Code de déontologie des avocats prévoit expressément que constitue un acte dérogatoire, en vertu du devoir d'intégrité, le fait d' « agir de façon à induire en erreur la partie adverse non représentée par avocat ou surprendre sa bonne foi ${ }^{114}$, la représentation loyale des intérêts de son client reste le premier mandat de l'avocat ${ }^{115}$. Son rôle d'officier de justice commande néanmoins sa collaboration «afin de favoriser l'accès à la justice [du] justiciable non représenté ${ }^{116}$. L'avocat devrait à cet égard utiliser un langage simple et clair, et même offrir une aide concrète dans la préparation du dossier et porter à la connaissance du tribunal les sources omises par le JNR ${ }^{117}$. Certains proposent de sensibiliser et de préparer les juristes beaucoup plus tôt à cette réalité, par l’offre de cours à la faculté de droit ${ }^{118}$, voire un programme de résidence inspiré du modèle existant en médecine ${ }^{119}$.

Alors que la nécessité d'assister les JNR apparaît comme une évidence, en raison du statut de l'avocat dans le système contradictoire et du rôle du tribunal au regard de l'administration de la justice, les juges semblent être considérés comme les mieux placés pour y parvenir ${ }^{120}$. Ils sont alors contraints de sortir de leur rôle passif pour compenser l'absence de représentation en prenant contrôle, du moins en partie, de l'instance ${ }^{121}$; le changement est si profond qu'il apparaît comme la «reconceptualisation » («re-

112 Philippe Samson, « Personnes non représentées - Une coexistence de devoirs à considérer » (2012) J du B 44 (8); Claude Duchesnay, « Se représenter seul » (2002) J du B 34 (13); Myriam Jézéquel, « Coût sociétal et conséquences pour les avocats et les magistrats - Le citoyen-plaideur sans avocat » (2005) J du B 37 (5); De Michele, Seul, supra note 3; Maria De Michele, « Comment traiter avec la partie non représentée? », dans Barreau du Québec, Congrès du Barreau : Un réseau d'avenir, juin 2011 , [en ligne :

www.caij.qc.ca/doctrine/congres_du_barreau/2011/2079/index.html\#_Toc293492600] (consulté le 6 septembre 2013) [Comment traiter].

113 Par ex. : Conseil canadien de la magistrature, supra note 110; Conseil de la magistrature du Québec, Rapport d'activité, Québec, 2006, p. 19; Robert Pidgeon, « L’avocat et la partie non représentée : jusqu’où le Tribunal peut-il repousser les limites déontologiques de l'avocat de la partie représentée? », dans Services de la formation permanente du Barreau du Québec, Développements récents en déontologie, droit professionnel et disciplinaire, Cowansville, Yvon Blais, 2006, pp 95-107; De Michele, La partie, supra note 11. Code de déontologie des avocats, RRQ, c B-1, r 3, art 3.02.01 (i).

115 Pidgeon, supra note 113. Voir également : Russel Engler, « Out of Sight and Out of Line: The Need for Regulation of Lawyers' Negotiation with Unrepresented Poor Persons » (1997) Cal L Rev 85, pp 79-170.

116 Ibid à la p 102.

117 Moorhead, supra note 38 à la p 411; ibid aux pp 101 et ss.

118 Macfarlane, The National, supra note 4 à la p 124.

119 Berenson, supra note 5 à la p 106.

120 De Michele, Comment traiter, supra note 112; Pearce, supra note 47; Zorza, An Overview, supra note 40 à la p 541; Jona Goldschmitt, « Strategies for Dealing with Self-Represented Litigants » (2008) NCL Rev 30, p 139.

121 MacFarlane, Opening, supra note 75; Moorhead, supra note 38; Englers, supra note 34 aux pp 2012-14; Branting, supra note 35 à la p 358; Buhai, supra note 10 aux pp 996 et 1003; Thompson, Le juge, supra note 20 à la p 3; Goldschmidt, supra note 32 à la p 609; Doherty, supra note 35 à la p 87; Zorza, The Disconnect, supra note 48. Selon le Conseil canadien de la magistrature, les juges doivent s'assurer que toutes les parties, représentées ou non, ont droit à l' " égalité d'accès au système judiciaire »; ils devront éventuellement prendre des « mesures non préjudiciables et positives des instances » : supra note 110 à la p 4. Les États-Unis ont adopté une mesure similaire : American Bar Association, Model Code of Judicial Conduct, 2011, Rule 2.2. La jurisprudence va également dans ce sens : par ex., Wagg c Canada, [2003] FCA 303; Desmarais c Longueuil (Ville de), 2010 QCCS 1686; Guenette c R, 2002 CanLII 7883 (QCCA). 
conceptualization ») du rôle judiciaire ${ }^{122}$. Le manque de balises claires pour encadrer cette transformation constitue la principale difficulté ${ }^{123}$. La pratique se développe conséquemment de façon hétérogène ${ }^{124}$, ce qui entraîne chez les JNR un sentiment d'incompréhension et d'abandon ${ }^{125}$.

La présence de JNR provoque inévitablement une modification de la dynamique judiciaire, et le fait de chercher à la rétablir a une incidence directe sur les tâches du personnel judiciaire, des avocats et des juges, qui se trouvent contraints de suppléer au travail du procureur manquant. L'obligation pour l'avocat de la partie adverse d'assister un JNR accroîtrait substantiellement ses frais de représentation ainsi que les frais judiciaires, en plus de menacer le lien de confiance avec son client ${ }^{126}$. Quant à la redéfinition du rôle judiciaire, elle perturbe à la fois les référents et les mécanismes de légitimation de l'institution, notamment l'apparence d'impartialité qui donne au tribunal l'autorité morale nécessaire pour trancher les litiges et pour que ses décisions soient appliquées ${ }^{127}$. Ces tentatives de modification des rôles traditionnels des acteurs du système juridique sont d'autant plus compliquées du fait que le manque de ressources humaines et financières permet difficilement d'envisager des changements majeurs et systémiques ${ }^{128}$. Certains proposent donc de faciliter le parcours judiciaire des JNR par le recours à des aménagements tels que la simplification de la procédure et l'utilisation de moyens alternatifs de résolution des conflits [MARC].

\section{Des aménagements internes au système juridique}

Règle générale, la doctrine présente la procédure comme le moyen de réduire les coûts, les délais et la complexité de la justice civile tout en assurant son humanisation et sa performance ${ }^{129}$. Depuis les années 1960, les différentes réformes de la procédure civile visent expressément à favoriser l'accès à la justice tout en préservant et en assurant la mise en œuvre du droit substantiel ${ }^{130}$. L'assouplissement de la procédure, mais également le recours à des MARC, dont les modes de fonctionnement sont plus flexibles et moins antagoniques, sont donc généralement présentés comme les moyens de rendre la justice plus accessible et moins éprouvante.

Il n'est pas surprenant de constater que la simplification de l'ensemble des étapes qui jalonnent le processus judiciaire apparaît comme le moyen le plus efficace de faciliter l'accès et la participation des

122 Zorza, An Overview, supra note 40 à la p 529.

123 Moorhead, supra note 38 à la p 410; Schwarz, supra note 32 à la p 662; Goldschmidt, supra note 32 à la p 616; Rebecca A. Albrecht, John M. Greacen, S. Bonnie Rose Hough et Richard Zorza, « Judicial Techniques for Cases Involving Self Represented Litigants » (2003) Judges J 24, pp 16-23; Deschênes c Valeurs mobilières Banque Laurentienne, 2010 QCCA 2137, paras 36 et ss.

124 Birnbaum, Bala et Bertrand, supra note 49 à la p 81. Dans un sondage mené dans le comté de Jackson (Missouri), les juges ont déclaré qu’ils aidaient les JNR « fréquemment » ou « toujours » (59 pour cent), « parfois » ou « nonfréquemment » (36 pour cent) ou « jamais » (18 pour cent) : Cynthia Cook, Self-Represented Litigants in Family Cases in Jackson County, Missouri, Institute for Court Management, Kansas City, 2007, p 35.

125 MacFarlane, Opening, supra note 75.

126 Birnbaum, Bala et Bertrand, ibid aux pp 108 et ss; Cameron et Kelly, supra note 10 aux pp 335-39; Langan, supra note 5 aux pp 11-12.

127 Moorhead, supra note 38 aux pp 408 et ss; Engler, supra note aux pp 2011 et ss; Macfarlane, supra note 3 aux pp 12425.

128 Zorza, An Overview, supra note 40 à la p 541.

129 Lafond, supra note 6.

130 Ibid; Hubert Reid, « Le nouveau Code de procédure civile : une étape? » (1965) C de D 6 (2), pp 29-37. 
JNR aux instances judiciaires ${ }^{131}$. Outre la simplification et la vulgarisation des formulaires, l'adaptation des règles de gestion des instances à la réalité des JNR est souvent présentée comme incontournable. Cette adaptation requiert néanmoins un rôle plus actif de la part du tribunal, comme la modification au besoin de quelques conventions ${ }^{132}$; la vulgarisation et l'assistance dans le décryptage du droit et la préparation des interrogatoires; la valorisation de la plaidoirie orale plutôt qu'écrite ou l'interprétation libérale des requêtes en faveur des $\mathrm{JNR}^{133}$. Les adaptations de cette nature imposent aux juges une posture compréhensive, par laquelle ils doivent s'assurer que les JNR sont bien en mesure de suivre et de participer au débat ${ }^{134}$. Dans cette optique, l'accompagnement actif des JNR, soit par un proche, soit par un «McKenzie friend ${ }^{135}$ », permettrait au tribunal de s'assurer de la représentation effective de ses intérêts.

Le recours à des MARC, et plus particulièrement à la médiation, est également présenté comme une des avenues facilitant la participation des JNR et surtout l'expression de leur point de vue ${ }^{136}$. Ce processus est en effet axé sur l'auto-détermination des parties et l'impartialité du médiateur, et non sur le travail de l'avocat ${ }^{137}$. En matière familiale, la médiation constitue de plus en plus le moyen privilégié, souvent imposé ${ }^{138}$, parfois en tant que service gratuit, en partie gratuit, ou dont les frais sont proportionnels au revenu $^{139}$.

En plus des mécanismes précités, certains auteurs proposent des stratégies globales comme la création de cours ou de chambres de la famille unifiées, où l'ensemble des services sociaux et juridiques serait facilement accessible ${ }^{140}$, ou encore de repenser l'architecture et l'organisation physique des palais de justice $^{141}$. Dans les deux cas, il s'agit d'assurer une plus grande cohérence entre les différents services et de réduire au minimum les démarches tout au long du processus judiciaire.

De tels aménagements, bien qu'ils facilitent jusqu'à un certain point le déroulement des instances, ne règlent en rien le problème du déséquilibre entre les parties. Les recherches démontrent au contraire que la simplification de la procédure n’est généralement pas suffisante pour permettre la réelle intégration

131 Richard Zorza, « Some First Thoughts on Court Simplification: The Key to Civil Access and Justice Transformation » (2013) Drake L Rev 61, pp 845-81.

132 Comme celles concernant l'ordre d'intervention des parties, les critères d'admissibilité de certains documents ou l'autorisation accordée aux JNR de s'adresser directement au juge.

133 Moorhead, supra note 38 à la p 411; Schwarz, supra note 32 à la p 668; Buhai, supra note 10 à la p 996. Voir également : Goldschmidt, supra note 32.

134 Macfarlane, The National, supra note 4 à la p 125.

135 Your McKenzie Friend: Family Law Help In \& Out of Court [en ligne : www.yourmckenziefriend.com] (consulté le 17 octobre 2013) :

A McKenzie Friend, traditionally, is a person that can sit with you in court and offer quiet advice, take notes, assist with documents and offer moral support. They may also help prior to court with applications and writing letters, discussing the legal proceedings with you, identifying the strengths and weaknesses of your case and in preparing your case for court.

136 Wissler, supra note 32 à la p 442.

137 Ibid à la p 435; Buhai, supra note 10 à la p 991.

138 Berenson, supra note 5 à la p 130.

139 Langan, supra note 6, paras 63 et ss. Au Québec, le ministère de la Justice assume depuis décembre 2012 les frais reliés à cinq heures de médiation familiale : Justice Québec, La médiation familiale - Négocier une entente équitable [en ligne : www.justice.gouv.qc.ca/francais/programmes/mediation/accueil.htm] (consulté le 18 octobre 2013).

140 Schwarz, supra note 32 à la p 661.

141 Zorza, The Self-Help, supra note 104 à la p 27. 
des JNR dans le débat judiciaire. De leur côté, les MARC peuvent provoquer, ou même exacerber, des rapports de force latents ou existants et être source de difficultés susceptibles d'avoir une incidence importante sur l'équité du processus ${ }^{142}$. L'absence d'intermédiaires tels que les avocats peut alors s'avérer dommageable, voire délétère ${ }^{143}$.

\section{CONCLUSION}

Récemment, la réception par la communauté juridique canadienne d'une étude sur la nonreprésentation a mis en évidence le malaise que le sujet suscite. La chercheure a fait face à des critiques virulentes concernant ses résultats, sa méthodologie - les participants à l'étude seraient «all crazy, angry people » - et même sa personne - traitée de «lawyer-bashing » ${ }^{144}$. C’est que son étude met en lumière le fait que les JNR entretiennent très majoritairement une impression négative de leur expérience judiciaire, notamment en raison de l'attitude de la magistrature à leur égard. Certains juges auraient une attitude hostile, critiquant le " choix » de se représenter soi-même, tenant des propos tels que «Don't bother coming back to court tomorrow if you're not wearing a tie » et préférant transiger avec l'avocat de la partie adverse ${ }^{145}$. Cette attitude est génératrice d'anxiété pour les JNR qui affirment ne pas se sentir à leur place, ne pas être pris au sérieux, voire être méprisés par les juges ${ }^{146}$. Ces résultats de recherche sont révélateurs du paradoxe selon lequel, alors que les JNR sont considérés comme des perturbateurs, qui imposent une pression indue sur le fonctionnement de la justice, la collaboration des acteurs judiciaires n'est pas constante et homogène. Lorsqu'elles demandent une participation active des avocats et des juges, les différentes solutions que propose la doctrine ne sont pas aisément mises en place $^{147}$.

Les avocats présentent une forte réticence à adapter leur pratique ${ }^{148}$ ou à accepter la présence des JNR. Un sondage mené auprès d'avocats ontariens exerçant en droit familial démontre qu'une forte majorité d'entre eux voient dans la non-représentation une situation compliquée, désagréable et problématique $^{149}$. Les JNR seraient incapables tant de fonctionner dans le système juridique que de communiquer efficacement avec eux ${ }^{150}$. Les avocats affirment éviter les contacts directs avec les JNR,

142 Macfarlane, The National, supra note 3 aux pp 73-75; Beck, Walsh, Ballard, Holtzworth-Munroe, Applegate et Putz, supra note 41 aux pp 631-32.

143 Macfarlane, ibid.

144 Blogue de Julie Macfarlane, Legitimate public concern - or lawyer-bashing? [en ligne : <http:// drjuliemacfarlane.wordpress.com/2013/07/03/legitimate-public-concern-or-lawyer-bashing/\#comments>] (consulté le 2 novembre 2013). Voir Schneider, supra note 13 aux pp 601-04.

146 Macfarlane, The National, supra note 3 aux pp 101-06. Voir également : Zimmerman et Tyler, supra note 19 aux pp 481 et ss.

147 Jona Goldschmitt, « The Pro Se Litigant Struggle for Access to Justice. Meeting the Challenge of Bench and Bar resistance » (2002) FCR 40 (1), pp 36-62; Smith et Stratford, supra note 109 à la p 215.

148 Blogue de Julie Macfarlane, It's the Culture, Stupid! Why lawyers aren't offering unbundled legal services [en ligne : http://drjuliemacfarlane.wordpress.com/2013/11/08/its-the-culture-stupid-why-lawyers-arent-offering-unbundled-legalservices/] (consulté le 8 novembre 2013).

149 Leurs commentaires sont unanimement péjoratifs : Birnbaum et Bala, supra note 3 aux pp 120 et ss.

150 Ibid à la p 110 et ss. 
les enjoignant de les contacter seulement par la poste ou par télécopieur ${ }^{151}$. Au Québec, les rares textes de doctrine portant sur les JNR, qui sont presque uniquement des formations à l'attention des avocats, en font un portrait peu reluisant.

De la même façon, une proportion importante de juges affirme qu'ils sont réticents à l'idée de déroger à leur rôle traditionnel afin d'assister les $\mathrm{JNR}^{152}$; ils opteraient généralement pour la posture passive classique $^{153}$. Il semblerait que l'attitude judiciaire puisse varier en fonction des motifs expliquant la non-représentation des justiciables : s'il s'agit d'un choix personnel et non d'une contrainte de nature financière, le tribunal aurait tendance à se cantonner à un rôle plus traditionnel ${ }^{154}$. Certains juges craindraient qu'une pratique judiciaire souple et clémente envers les JNR encourage la nonreprésentation $^{155}$, mais l'explication la plus souvent avancée touche aux effets préjudiciables d'un certain activisme judiciaire sur les prémisses fonctionnelles du système juridique ${ }^{156}$. Maintenir le tribunal dans son rôle serait un moyen de préserver les conditions normales du procès, notamment l'égalité des parties et l'apparence d'impartialité. Il faut cependant savoir que si, pour les avocats, l'assistance offerte par les juges se situe à la frontière du conseil juridique - et ils questionnent l'effet de cette pratique sur l'équité des procès $^{157}$ - les profanes n'ont pas nécessairement la même interprétation $^{158}$.

La passivité du tribunal peut pourtant mener à l'impossibilité pour les JNR de faire valoir leurs droits $^{159}$, sans pour autant garantir l'impartialité du tribunal ${ }^{160}$. Il s’agirait alors de « conserver la longue tradition des tribunaux supérieurs élitistes [...], soit l'accès limité aux personnes peu fortunées, et peu d'égard pour l'issue du procès » ${ }^{161}$, ce qui apparaît de moins en moins viable dans une situation où le nombre de JNR ne cesse d'augmenter. Dans de telles circonstances, l'image de la justice ne peut que se

151 Birnbaum et Bala, supra note 3 à la p 121.

152 Cameron et Kelly, supra note 10 à la p 329; Englers, supra note 34 à la p 2014; William Hornsby, Cynthia Gray et John Greacen, « Self-Represented Litigants and the Ethics of Lawyers, Judges, and Court Staff », dans Hannaford-Agor, supra note 36 à la p 73. Une étude menée dans le comté de Jackson (Missouri) a démontré que 45 pour cent des juges sont contre une quelconque assistance aux JNR, alors que 46 pour cent des avocats se disent en faveur d'une telle assistance : Cook, supra note 124.

153 Englers, supra note 34 aux pp 2014-18; Langan, supra note 6, paras 29-33; D. A. Rollie Thompson, « No Lawyer: Institutional Coping with the Self-Represented » (2002) Can Fam LQ 19, p 474 et 485 [No Lawyer]; Blishen, supra note 60 à la p 124; Moorhead, supra note 38 à la p 410; Richardson, Sourdin et Wallace, supra note 5 à la p 18.

154 Englers, ibid aux pp 2014-18; Langan, ibid, paras 29-33; Thompson, ibid aux pp 474 et 485; Blishen, supra note 60 à la p 124; Moorhead, supra note 38 à la p 410.

155 Englers, ibid à la p 2015, se référant à Jona Goldschmidt, « How Are Judges and Courts Coping with Pro Se Litigants?: Results from a Survey of Judges and Court Managers passim », p 18 (document non publié); Moorhead, ibid à la p 410.

156 Cameron et Kelly, supra note 10 à la p 334.

157 Birnbaum et Bala, supra note 4 à la p 120. Voir également : Jona Goldschmitt et Loretta Stalans, « Lawyers' Perceptions of the Fairness of Judicial Assistance to Self-Represented Litigants » (2012) Windsor YB Access Just 30, p 139.

158 Jona Goldschmitt, « Judicial Ethics and Assistance to Self-Represented Litigants » (2007) Just Sys J 28 , p 326.

159 Branting, supra note 35 à la p 358.

160 Zorza, The Disconnect, supra note 48.

161 Thompson, Le juge, supra note 20 à la p 5. 
dégrader ${ }^{162}$, ce qui est à prendre au sérieux dans un contexte où la fréquentation des tribunaux civils est en chute libre ${ }^{163}$.

Quelques auteurs proposent une piste de réflexion qui semble prometteuse. Il s'agit essentiellement de restructurer le système judiciaire autour de son objectif premier : le règlement juste et équitable des conflits $^{164}$. Autrement dit, il ne serait plus simplement question d'assurer l'égalité formelle des parties, mais de tenir compte des éléments susceptibles d'avoir un effet sur les conditions d'exercice des droits $^{165}$, ce qui implique un réel bouleversement de la raison juridique. Une transformation aussi radicale n'est évidemment envisageable qu'avec le concours actif et concerté des organes professionnels (barreaux et conseils de la magistrature) et des facultés de droit ${ }^{166}$.

La remise en question de certaines prémisses du système juridique - notamment la procédure contradictoire, l'impartialité et l'apparence d'impartialité des juges - permettrait de repenser les conditions de l'accès à la justice, non en termes formels mais plutôt par rapport à ce qui arrive concrètement aux personnes dans leurs contacts avec l'institution judiciaire ${ }^{167}$. Il s'agirait de reconnaître qu'au-delà du litige subsiste une histoire, inscrite dans un contexte social et interpersonnel que l'on ne peut négliger. Ce retournement de perspective exige un réel engagement des acteurs judiciaires auprès des justiciables à toutes les étapes de l'élaboration d'un règlement, et de veiller à ce que le processus soit clair et que tous les faits importants aient pu être portés à la connaissance du tribunal ${ }^{168}$.

Un tel engagement ne sera possible qu'avec l'émancipation du droit et de la justice par rapport aux lois du marché ${ }^{169}$ par lesquelles les tribunaux ne sont qu'un «club privé », le " domaine privilégié de citoyens eux-mêmes privilégiés ${ }^{170}$ ». " Given this strong influence, the use of the market as the primary mechanism for distributing legal services guarantees significantly unequal justice under law ${ }^{171}$. » Tant que la qualité des services juridiques - et donc de la représentation - dépendra de la capacité de payer, faciliter l'accès à des avocats, notamment en rehaussant les plafonds d'admissibilité à l'aide juridique, ne permettra pas de régler le problème de la transposition des inégalités sociales devant les tribunaux ${ }^{172}$. Il faut dire que, depuis que les tribunaux existent, les services juridiques sont payants, et que si certains

162 Seulement 63 pour cent des québécois feraient « très ou assez » confiance au système judiciaire : Lafond, supra note 6, p 31.

163 Ibid aux pp 33 et ss.

164 Russell Engler, «Ethic in Transition: Unrepresented Litigants and the Changing Judicial Role » (2008) Notre Dame JL Ethics \& Pub Pol'y 22, p 385.

165 Gregori Peces-Barba Martinez, Théorie générale des droits fondamentaux, Paris, LGDJ, 2003, pp 259 et ss; Ronald Dworkin, La vertu souveraine, Bruxelles, Bruylant, 2007.

166 Macfarlane, The National, supra note 4 aux pp 127-28.

167 Zimmerman et Tyler, supra note 19 à la p 480.

168 Zorza, The Disconnect, supra note 48 aux pp 426 et ss.

169 Stephen Tomsen, « Legal Services and Neo Liberalism in an Unequal Legal Order » (2008) FJLR 10, pp 609-20.

170 Pierre Noreau, « Accès à la justice et démocratie en panne : constats, analyses et projections », dans Pierre Noreau (dir.), Révolutionner la justice. Constats, mutations et perspectives, Montréal, Thémis, 2010, pp 39-40.

171 Pearce, supra note 47 à la p 972.

172 Ibid à la p 970. Voir également : Hélène Tessier, « Pauvreté et droit à l’égalité : égalité de principe ou égalité de fait? », dans Service de la formation permanente du Barreau du Québec, Développements récents en droit administratif, Cowansville, Yvon Blais, 1998, pp 47 et 65; Jean Hétu et Herbert Marx, « Les défavorisés, le Code civil et les juges » (1976) R D McGill 22, p 367; Henri Brun et André Binette, «L'interprétation judiciaire de la condition sociale, motif de discrimination prohibé par la Charte des droits du Québec » (1981) C de D 22, pp 681-94. 
d'entre eux ont été rendus disponibles pour les plus pauvres, ce fut longtemps conformément à une logique de charité ${ }^{173}$. Les services d'aide juridique ont bien été établis sur d'autres bases par les Étatsprovidence ${ }^{174}$, mais la diminution dramatique de leur accessibilité est corolaire à la recrudescence des services de bienfaisance ${ }^{175}$ que certains voudraient voir devenir obligatoires ${ }^{176}$. Alors que l'accès à la justice civile apparaît comme la condition d'une forme d'égalité sociale ${ }^{177}$ ou le moyen d'actualisation d'un statut citoyen ${ }^{178}$, il s'agit de repenser la justice en tant que service public au même titre que la santé ou l'éducation ${ }^{179}$. Elle ne serait ainsi plus «l'apanage des juges et des avocats ${ }^{180}$ », mais une «responsabilité sociétale ${ }^{181} »$. Elle ne serait plus une institution mythique, mais une institution citoyenne ancrée dans une communauté et contribuant positivement à sa qualité de vie ${ }^{182}$.

173 Isabel Fanlo Cortès, « Justice for the Poor in the Hands of the Lawyers? Some Remarks on Access to Courts and Legal Aid Models » (2008) Cuadernos Constitucionales de la Cátedra Fadrique Furió Ceriol 64/65, pp 51-52; Richard Abel, " Law without Politics: Legal Aid under Advanced Capitalism » (1985) UCLA L Rev, pp 474-642.

174 Soulignons tout de même que le fait de parler d' « aide juridique » plutôt que d' « assurance juridique » est révélateur d'une conception plus près de la charité que de la solidarité.

175 Association du Barreau canadien, «Tension à la frontière » : services pro bono et aide juridique, Ottawa, 2012; Richard Abel, « The Paradoxes of Pro Bono » (2010) Fordham L Rev 78 (5), pp 2443-50.

176 Richard Devlin, « Breach of Contract: The New Economy, Access to Justice and the Ethical Responsibilities of the Legal Profession » (2002) Dalhousie LJ 25, pp 335-75.

177 Jason M. Solomon, « Civil recourse as social equality » (2011) Fla St U L Rev 39, pp 243-72.

178 Hilary Sommerlad, «Some Reflections on the Relationship between Citizenship, Access to Justice, and the Reform of Legal Aid » (2008) J Law \& Soc 31 (3), pp 345-68.

179 Soulignons par ex. l'initiative du ministère de la Justice du Nouveau-Brunswick, qui finance les activités du Service public d'éducation et d'information juridiques du Nouveau-Brunswick, [en ligne : www.legal-infolegale.nb.ca/fr/funding] (consulté le 8 novembre 2013).

180 Marie Deschamps, « L’accès à la justice, l’affaire de chacun » (2009) C de D 50 (1), p 258.

181 Jean-Guy Belley, «Une justice de la seconde modernité : proposition de principes généraux pour le prochain Code de procédure civile » (2001) R D McGill 46, p 371.

182 Deschamps, supra note 180 à la p 251. 